\title{
Cervical pregnancy managed by local excision
}

\author{
S. A. FARGHALY \\ M.B., Ch.B., D.G.O.
}

\author{
J. G. Mathie \\ F.R.C.S., F.R.C.O.G.
}

Department of Gynaecology and Obstetrics, Essex County Hospital, Colchester

\begin{abstract}
Summary
A patient with cervical pregnancy is described who presented with a history of 7 weeks' amenorrhoea and painless vaginal bleeding. Examination revealed an intracervical mass, the internal os was closed and the external os was dilated. The cervical pregnancy is rare and is discussed.
\end{abstract}

\section{Introduction}

Studdiford (1969) defined cervical pregnancy as implantation and development of the fertilized ovum within the cervical structure without involving the corpus uteri. This rare ectopic gestation has been reported in the literature infrequently and the time incidence is not known. Dees (1966) found the incidence of cervical pregnancy to be about 1:18000 pregnancies.

\section{Case report}

A 26-year-old married primigravida with 7 weeks' amenorrhoea was admitted as an emergency complaining of painless vaginal bleeding for about 2 days. Two weeks before admission she had had a similar bleeding which she thought was a period. There was no history of abdominal pain.

Her general condition was good. BP and pulse were normal. Abdominal examination showed no obvious abnormality. Vaginal examination showed that she was bleeding heavily. The findings on digital examination suggested that there was a mass within the cervical canal. The uterus was normal with no adnexal tmasses. Speculum examination confirmed the presence of a mass resembling products of conception in the cervical os. The patient was referred for surgery.

At operation an intracervical mass about $8 \mathrm{~cm}$ in diameter was found. It was protruding through the dilated external os, the cervix was thinned, and the internal os was closed. There was bleeding from this mass. The mass was carefully dissected free from the cervix and sent for histological examination. Three biopsies were taken from the cervix. The uterine cavity was $8 \mathrm{~cm}$ and curettage was carried out. Endometrial curettings and cervical biopsies were sent for histological examination and the vagina was firmly packed. Postoperatively the patient made good progress and was discharged from the hospital on the 4th postoperative day. She was well when seen 6 weeks later and the cervix had completely healed. Histology of the endometrial curettings showed endometrium in late proliferative phase, the cervical biopsies showed loss of epithelium and replacement by decidua with some recognizable cytotrophoblast, and the presence of trophoblastic tissue was confirmed in the cervical tissues.

\section{Discussion}

This case history conforms to clinical criteria suggested by Duckman (1951) for the diagnosis of cervical pregnancy. The following criteria (a) a dilated, thin-walled cervical canal containing histological evidence of gestation, (b) a patulous os, (c) a small and firm corpus luteum with normal size internal os.

The general course of a cervical pregnancy is early abortion. Pisarski (1960) has reported a case of cervical pregnancy which resulted in delivery of a living infant.

Flanagan and Walsh (1954) suggested that local excision is feasible owing to the decidual necrosis and thrombosis of the end vessels of the placenta after the death of the pregnancy.

Other procedures such as packing the cervix, and amputation of the cervix and leaving the placenta have been suggested.

In the majority of cases reported in the literature, particularly those which had progressed beyond 8 weeks' gestation, the ultimate method of controlling the bleeding was by means of abdominal hysterectomy. Punch biopsy of the cervix is sometimes necessary to differentiate between cervical carcinoma and cervical pregnancy. The risk of a further cervical pregnancy is unknown and the occurrence of cervical pregnancy would not affect the competency of the internal os.

\section{References}

DeEs, H.C. (1966) Cervical pregnancy associated with uterine leiomyomas. Southern Medical Journal, 59, 900.

DuCkman, S. (1951) Cervical pregnancy. American Journal of Obstetrics and Gynecology, 62, 1381.

Flanagan, J.F. \& Walsh, G.R. (1954) Cervical pregnancy, report of a case. Obstetrics and Gynecology, 4, 511.

PISARSKI, T.S. (1960) Cervical pregnancy. Journal of Obstetrics and Gynaecology of the British Commonwealth, 67, 759.

STUDDIFord, W.E. (1969) Cervical pregnancy. American Journal of Obstetrics and Gynecology, 105, 282. 